\title{
Minification of fundus optical coherence tomographic images in gas-filled eye
}

Toshifumi Yamashita ${ }^{\dagger}$, Hiroto Terasaki ${ }^{* \dagger}$ and Taiji Sakamoto

\begin{abstract}
Background: Optical coherence tomography $(\mathrm{OCT})$ is being used increasingly to evaluate and manage a variety of retinal diseases, but not much is known about the minification of the OCT images in gas-filled eyes. The purpose of this study was to investigate the effect of gas-filled eyes on the size of the OCT images.

Methods: This was retrospective case series of 81 consecutive eyes of 79 patients who had macular hole surgery between April 2012 and September 2014. Images of the optic disc were taken with a spectral domain-OCT instrument 2 days after surgery in gas-filled, pseudophakic eyes and from the same eyes but fluid-filled one month after the surgery. The vertical length, horizontal width, and the area of the optic disc were measured in the OCT images.
\end{abstract}

Results: Clear images were obtained from 50 eyes of 49 patients (mean age $66.4 \pm 5.9$ years). The mean vertical length and mean horizontal width of the optic disc in the gas-filled eyes were about $25 \%$ shorter than that of fluid-filled eyes (vertical, $1213.8 \pm 170.5$ and $1650.6 \pm 195.9 \mu \mathrm{m}, P<0.01$; horizontal, $1169.4 \pm 143.1$ and $1526.4 \pm$ $219.9 \mu \mathrm{m}, P<0.01)$. The mean area of the optic disc was $1.12 \pm 0.34 \mathrm{~mm}^{2}$ in gas-filled eyes which was significantly smaller than that in fluid-filled eyes $\left(1.88 \pm 0.37 \mathrm{~mm}^{2}\right)$ by $40.4 \%$.

Conclusions: The fundus images of gas-filled eyes are significantly smaller than that in the same fluid-filled eyes. The minification of the OCT images should be considered when analyzing images obtained from gas-filled eyes.

Trial registration: Trial registration number: UMIN000007517. Date of registration: 3/21/2012.

Keywords: Macular hole, SD-OCT, Gas-filled eye

\section{Background}

Recent improvements in optical coherence tomography (OCT) has made it a useful and indispensable tool for clinical ophthalmology [1-4]. OCT is used not only for diagnosis but also for monitoring the effectiveness of therapy [5, 6]. Recently, OCT instruments that allowed photographing the fundus of gas-filled eyes with a macular hole $(\mathrm{MH})$ has become commercially available [7-13]. This has allowed the collection of important clinical information for the diagnosis and treatment of MHs. Clear OCT images of the macular configuration was obtained from gas-filled eyes that provided important information that could be used to determine the duration of the face-down position

\footnotetext{
* Correspondence: teracchi@m2.kufm.kagoshima-u.ac.jp

${ }^{\dagger}$ Equal contributors

Department of Ophthalmology, Kagoshima University Graduate School of Medical and Dental Sciences, Kagoshima, Japan
}

needed after MH surgery [9-12]. In addition, information of the closing process of a $\mathrm{MH}$ after surgery in the very early phase under gas provided information on this disease process $[8,11,12]$.

The values of the measured parameters of the images recorded are strongly affected by the intraocular media, such as gas, oil, and saline solutions. A recent study showed that the fundus images recorded by a wide-field scanning ophthalmoscope was minified in gas-filled eyes [14].

It is essential to know the effect of the intraocular media on the OCT images before the general application of this method in clinical studies and on patients. To the best of our knowledge, there has not been a study on the minifying effect of the OCT images recorded in gas-filled eyes. Thus, the purpose of this study was to determine the effect of intraocular gas on the size of the images recorded by OCT. 


\section{Methods}

The procedures used in this study were approved by the Institutional Review Board of the Kagoshima University Hospital, and they conformed to the tenets of the 1989 Declaration of Helsinki. This study was registered with the University Hospital Medical Network (UMIN)-clinical trials registry and the registration number was UMIN000007517. A detailed explanation of the procedures was given to the patients, and a written informed consent was obtained from all. The patients also agreed to allow us to use the data for future analyses and publications.

This was a retrospective consecutive case series that included 81 consecutive eyes of 79 patients treated for a $\mathrm{MH}$ at the Kagoshima University Hospital between April 2012 and September 2014. The surgery consisted of standard pars plana vitrectomy with either a 23-gauge or 25 -gauge system as described in detail [11, 12]. Briefly, the internal limiting membrane was peeled after core vitrectomy and a separation of the posterior hyaloid membrane. After the surgery, the contents of the vitreous cavity were exchanged with non-expansile $20 \%$ sulfur hexafluoride (SF6). Phakic patients older than 50 years underwent cataract surgery with implantation of an intraocular lens before the vitrectomy.

\section{Optical coherence tomography}

Images of the optic disc were taken with a spectral domain OCT instrument 2 days after surgery in gas-filled eyes and from the same eyes one month after the surgery when the gas had been absorbed and the cavity was fluid-filled. We analyzed only the high-quality OCT images of gas-filled eye as reported elsewhere [11, 12]. During the follow-up period, we recorded images not only of the macular area but also of the optic disc with the Cirrus HD-OCT (Carl Zeiss Meditec, Dublin, Calif., USA). For this, the focus was adjusted to -20 diopters and scanning was performed. The focus on the fundus and B scan images was always adjusted simultaneously to get the best focused image. A detailed description of the methods was reported in our previous publications $[11,12]$. From our experience, a Cirrus HD-OCT is the best instrument to obtain clear images of gas-filled eyes compared to other OCT instruments because of the differences in the range of focus, recordings can be made with or without the auto-focus function, and ability to alter the spacing of each slice in the B-scan recordings.

The optic disc area was scanned with the optic disc cube $200 \times 200$ protocol. All SD-OCT examinations were performed with the patients sitting in an upright position. Most OCT instruments including the Cirrus HD-OCT have an embedded function to assess the image intensity and quality, which is expressed as signal strength in the Cirrus HD-OCT. Lower signal-to-noise ratios indicate poor image quality, and these images should not be used to assess the retina especially in older subjects. So, images with a signal strength $<5$ were excluded.

\section{Measurements of vertical lengths and horizontal widths and areas of optic disc}

The vertical lengths and horizontal widths of the optic disc were measured semi-automatically with a program built-into the Cirrus OCT. In the optic disc cube $200 \times$ 200 protocol, a $6 \times 6 \mathrm{~mm}$ area was recorded with 200 consecutive scans. Then, the size of each slice is $30 \mu \mathrm{m}$ $(6000 \mu \mathrm{m} / 200$ scans $=30 \mu \mathrm{m})$. The length of the optic disc in the images was calculated as (Fig. 1):

$$
\text { length of optic disc }=\text { Number of scan X } 30 \mu \mathrm{m} \text {. }
$$

The optic disc area was calculated by a software embedded in the OCT instrument.

\section{Statistical analyses}

All statistical analyses were performed with the SPSS program for Windows (SPSS Inc., IBM, Somers, New York, USA). Wilcoxon signed-rank tests were used to determine the significance of differences in the data. A $P$ value $<0.05$ was taken to be statistically significant.

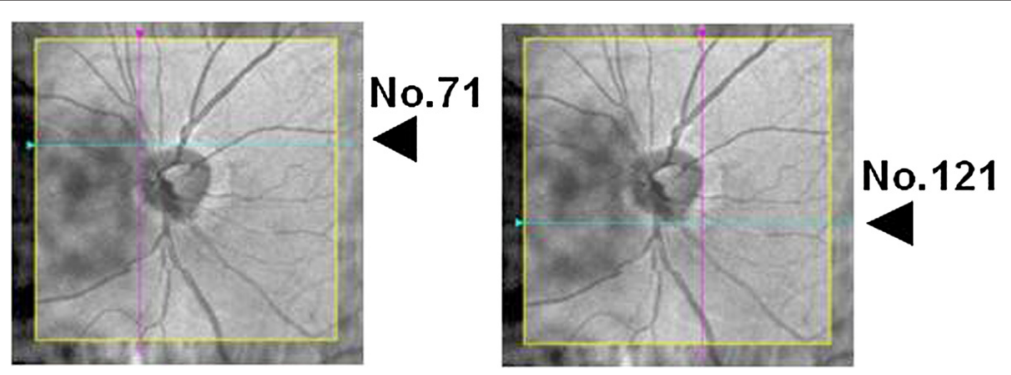

Fig. 1 Measurement of the vertical length and horizontal width of the optic disc. The size of optic disc was measured in the Optic Disc Cube $(200 \times 200)$ images of the Cirrus HD-OCT. Because the spacing of each slice is $30 \mu \mathrm{m}$, the length of the optic disc was calculated as: Length of optic disc $=$ Number of scan $\times 30 \mu \mathrm{m}$. Because the number of scans is 50 (i.e.,121-71) in this case, the vertical length of optic disc is $1500 \mu \mathrm{m}$ 


\section{Results}

Eighty-one consecutive eyes of 79 patients that had undergone vitrectomy for a $\mathrm{MH}$ were studied. Thirtyone eyes of 30 patients were excluded because the quality of the images was $<5$. Thus, 50 eyes of 49 cases were studied of which 27 were women and 22 were men. Their mean \pm SD age was $66.4 \pm 5.9$ years with a range of 56 to 85 years. Forty-seven eyes were phakic preoperatively and 3 eyes were pseudophakic. The 47 eyes underwent phacoemulsification and lens implantation before the vitrectomy, and the 3 pseudophakic eyes had vitrectomy alone. As a result, all OCT images taken were from pseudophakic eyes.

\section{Vertical length, horizontal width, and area of optic disc}

The mean vertical length of the optic disc was $1213.8 \pm$ $170.5 \mu \mathrm{m}$ two days after surgery when the eye was gasfilled and $1650.6 \pm 195.9 \mu \mathrm{m}$ at one month after the surgery when the eye was fluid-filled. The mean horizontal width of the optic disc was $1169.4 \pm 143.1 \mu \mathrm{m}$ 2 days after surgery when the eye was gas-filled and $1526.4 \pm 219.9 \mu \mathrm{m}$ one month after the surgery when the eye was fluid-filled. Thus, the vertical length was shorter by $26.5 \%$ and the horizontal width by $23.5 \%$. The vertical length and horizontal width of the optic disc in the gas-filled eye were significantly shorter than that in the fluid-filled eyes $(P<0.01$, Fig. $2 \mathrm{a}-\mathrm{b})$.

The mean area of the optic disc was $1.12 \pm$ $0.34 \mathrm{~mm}^{2}$ in the gas-filled eye which was significantly smaller than that in the fluid-filled eyes at $1.88 \pm$ $0.37 \mathrm{~mm}^{2}(P<0.01)$. The reduction was by $40.4 \%$ (Fig. 2c). Representative images including one with an unclosed $\mathrm{MH}$ and with the optic disc are shown in Fig. 3 and Additional file 1.

\section{Discussion}

Our results showed that the images from gas-filled eyes were about $25 \%$ smaller in both the vertical and horizontal directions and about $40 \%$ smaller in the area than the images obtained from the same eyes after the gas was absorbed and the vitreous cavity was fluid-filled. Images of gas-filled eyes by the ultra-wide-field scanning ophthalmoscope Optos 200Tx (Optos, Marlborough, MA, USA) were also reported to be minified [14]. Meyer et al reported that the images obtained with a panfunduscopic lens were $30 \%$ smaller in gas-filled eye than in eyes without gas or air [15]. They did not present detailed methods for the measurements and calculations. However, the degree of reduction is comparable to our findings. Since the measurements in our patients were made by a built-in program of the Cirrus HD-OCT, this method was more objective.

The reduction in the image size can lead to an error in the size of an unclosed $\mathrm{MH}$ in gas-filled eye. For example, the image of the $\mathrm{MH}$ in the gas-filled eye was smaller than that in the same eye without gas (Fig. 3a). The actual length of the optic disc in the OCT images of gas-filled eyes would be expected to be larger when the gas is replaced by fluid. The measurements can be corrected by multiplying the values by 1.3 because the length of optic disc in gas-filled eyes was about $25 \%$ smaller. A MH closure might occasionally be overlooked in gas-filled eye in the original width of each scan of $0.25 \mathrm{~mm}$ in the 5-line raster scan. A width of $0.025 \mathrm{~mm}$,
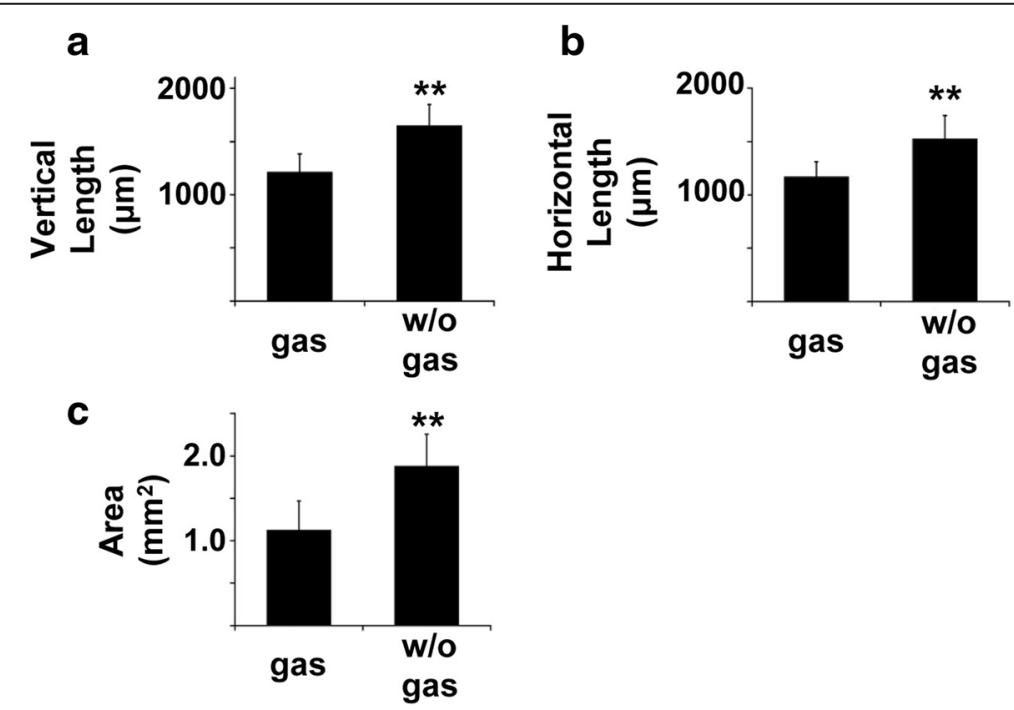
same eyes after the gas had been absorbed and the eye was fluid-filled ( $\mathbf{a}, P<0.01)$. The horizontal width was $26.5 \%$ narrower in gas-filled eyes than after the gas is absorbed and the eye is fluid-filled (b, $P<0.01)$. The area of the optic disc of gas-filled eye was significantly smaller than eyes after the gas was absorbed by $40.4 \%(\mathbf{c}, P<0.01)$ 


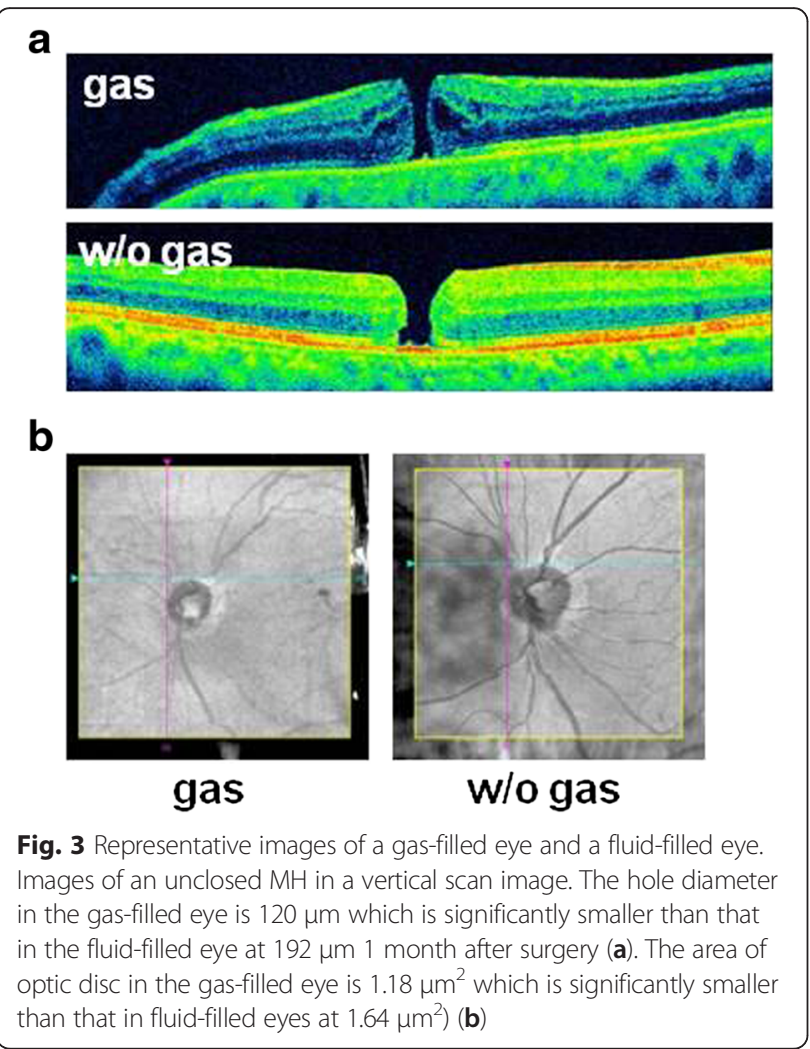

which is narrowest setting in this protocol, should be suitable for confirming a closure of a $\mathrm{MH}$. This can also occur with a $0.075 \mathrm{~mm}$ spacing (Fig. 4).

There are several reports studying the OCT images in silicone oil-filled eyes showing the very early processes of $\mathrm{MH}$ closure after vitrectomy [16-18]. The results indicated that the disappearance of the fluid-cuff and peri-MH cysts are important signs of $\mathrm{MH}$ closure. However, a detailed description of the changes that occur during $\mathrm{MH}$ closure in gas-filled eyes had not been published which is important to determine the choice of the most effective treatment. Kukushima et al recently reported on the very early stage of $\mathrm{MH}$ closure soon after vitrectomy with SF6 gas tamponade using OCT imaging in the same way we did [8]. They reported the MH closure occured in 3 distinct steps. In step 1 , the torn and separated edges of the photoreceptor layer which forms the minimum $\mathrm{MH}$ diameter preoperatively, formed a kissing configuration from as early as 20 min after the vitrectomy up until day 1 . In step 2 , the residual $\mathrm{MH}$ above the connected photoreceptor layer closes. Finally in step 3, the subretinal fluid below the connected photoreceptor layer is absorbed and the $\mathrm{MH}$ is completely closed [8]. The minification of the OCT images in gas-filled eyes should be noted in investigations of OCT images of gas-filled eyes or comparison of dynamics of $\mathrm{MH}$ closure between silicon oil tamponade and SF6 tamponade.

There are limitations in this study. First, this was a retrospective study and potential sampling bias cannot be eliminated. Second, the eyes were limited to those with a $\mathrm{MH}$ treated with $20 \%$ SF6 gas. Third, taking high-quality images of optic disc in gas-filled eyes were relatively difficult compared to that of B-scan as reported $[11,12]$ probably because of the reflection or non-specific noises. Fourth, although this study excluded highly myopic eyes [11, 12], ocular magnification might affect our results and the minification effect in gas-filled eye on the retinal thickness were not determined [19].

\section{Conclusions}

The optic discs in SD-OCT images of gas-filled eye are about $25 \%$ shorter in the vertical and horizontal lengths, and $40 \%$ smaller in area than the size of the same eyes after the gas is absorbed. This information is essential for correct evaluation of the status of the fundus images in gas-filled eyes.

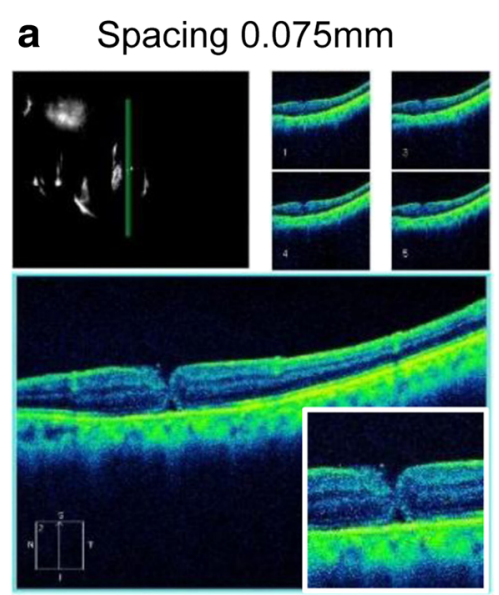

b Spacing $0.025 \mathrm{~mm}$

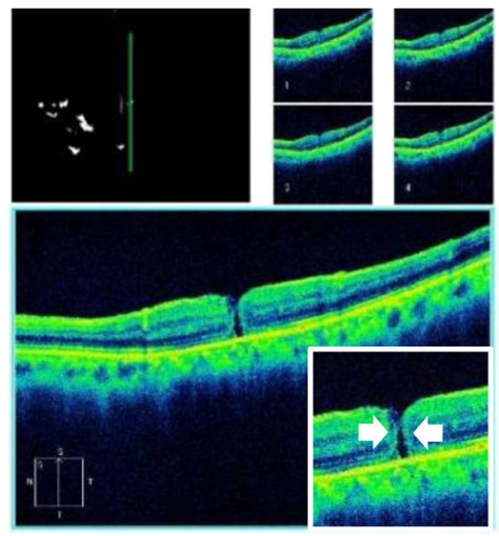

Fig. 4 Appearance of MH with scan spacing of $0.075 \mathrm{~mm}$ and $0.025 \mathrm{~mm} 2$ days after vitrectomy. The MH appears approximately closed in images of $0.075 \mathrm{~mm}$ space scan (a) but clearly not closed in $0.025 \mathrm{~mm}$ space scan (b, white arrows) 


\section{Additional file}

Additional file 1: Representative images of optic disc in a gas-filled eye and a fluid-filled eye. (JPG $154 \mathrm{~kb}$ )

\section{Abbreviations}

$\mathrm{MH}$, macular hole; $\mathrm{OCT}$, optical coherence tomography; SF6, sulfur hexafluoride

\section{Acknowledgments}

The authors thank Professor Duco Hamasaki of Bascom Palmer Eye Institute, University of Miami, FL, for providing critical discussions and suggestions to our study and editing of the final manuscript.

\section{Funding}

This study was in part sponsored by a grant from the Research Committee on Chorioretinal Degeneration and Optic Atrophy, Ministry of Health, Labor, and Welfare, Tokyo, Japan; and by a Grant-in-Aid for Scientific Research from the Ministry of Education, Science, and Culture of the Japanese Government, Tokyo, Japan.

\section{Availability of data and materials}

The date in this study will be shared upon request.

\section{Authors' contributions}

TY and HT contributed to planning the study, and data collection and analysis. HT and TS drafted the manuscript. All authors read and approved the manuscript.

\section{Competing interests}

The authors declare that they have no competing interests.

\section{Consent for publication}

The patients agreed to allow us to use the data for future analyses and publications.

\section{Ethics approval and consent to participate}

The procedures used in this study were approved by the Institutional Review Board of the Kagoshima University Hospital, and they conformed to the tenets of the 1989 Declaration of Helsinki. A detailed explanation of the procedures was given to the patients, and a written informed consent was obtained from all.

Received: 9 February 2016 Accepted: 18 July 2016

Published online: 26 July 2016

\section{References}

1. Sakata LM, Deleon-Ortega J, Sakata V, Girkin CA. Optical coherence tomography of the retina and optic nerve - a review. Clin Experiment Ophthalmol. 2009:37(1):90-9.

2. Terasaki H, Shirasawa M, Yamashita T, Yamakiri K, Sonoda S, Sakamoto T. Comparison of foveal microstructure imaging with different spectral domain optical coherence tomography machines. Ophthalmology. 2012;119(11):2319-27.

3. Wolf S, Wolf-Schnurrbusch U. Spectral-domain optical coherence tomography use in macular diseases: a review. Ophthalmologica. 2010;224(6):333-40.

4. Yamashita T, Shirasawa M, Arimura N, Terasaki H, Sakamoto T. Repeatability and reproducibility of subfoveal choroidal thickness in normal eyes of Japanese using different SD-OCT devices. Invest Ophthalmol Vis Sci. 2012;53(3):1102-7.

5. Hee MR, Puliafito CA, Wong C, Duker JS, Reichel E, Schuman JS, et al. Optical coherence tomography of macular holes. Ophthalmology. 1995; 102(5):748-56.

6. Oh J, Smiddy WE, Flynn Jr HW, Gregori G, Lujan B. Photoreceptor inner/outer segment defect imaging by spectral domain OCT and visual prognosis after macular hole surgery. Invest Ophthalmol Vis Sci. 2010;51(3):1651-8.

7. Eckardt C, Eckert T, Eckardt U, Porkert U, Gesser C. Macular hole surgery with air tamponade and optical coherence tomography-based duration of facedown positioning. Retina. 2008;28(8):1087-96.

8. Kikushima W, Imai A, Toriyama Y, Hirano T, Murata T, Ishibashi T. Dynamics of macular hole closure in gas-filled eyes within $24 \mathrm{~h}$ of surgery observed with swept source optical coherence tomography. Ophthalmic Res. 2015; 53(1):48-54
9. Masuyama K, Yamakiri K, Arimura N, Sonoda Y, Doi N, Sakamoto T. Posturing time after macular hole surgery modified by optical coherence tomography images: a pilot study. Am J Ophthalmol. 2009;147(3):481-8. e2.

10. Shah SP, Manjunath V, Rogers AH, Baumal CR, Reichel E, Duker JS. Optical coherence tomography-guided facedown positioning for macular hole surgery. Retina. 2013;33(2):356-62.

11. Yamashita T, Kawano H, Sonoda Y, Yamakiri K, Sakamoto T. Early imaging of macular hole closure: a diagnostic technique and its quality for gas-filled eyes with spectral domain optical coherence tomography. Ophthalmologica. 2013;229(1):43-9.

12. Yamashita T, Sakamoto T, Sonoda S, Yamakiri K, Otsuka H, Hisatomi T, et al. Individualized, spectral domain-optical coherence tomography-guided facedown posturing after macular hole surgery: minimizing treatment burden and maximizing outcome. Retina. 2014;34(7):1367-75.

13. Chow DR, Chaudhary KM. Optical coherence tomography-based positioning regimen for macular hole surgery. Retina. 2015;35(5):899-907.

14. Oishi A, Hidaka J, Yoshimura N. Quantification of the image obtained with a wide-field scanning ophthalmoscope. Invest Ophthalmol Vis Sci. 2014;55(4):2424-31.

15. Meyers SM, Nothnagel AF. Panfunduscope lens for laser photocoagulation in gas-filled pseudophakic or aphakic eyes. Arch Ophthalmol. 1985;103(8): 1109-10.

16. Hotta K. Early postoperative macular features determined by optical coherence tomography after idiopathic macular hole surgery with silicone oil tamponade. Ophthalmic Surg Lasers Imaging. 2005;36(5):426-31.

17. Jumper JM, Gallemore RP, McCuen 2nd BW, Toth CA. Features of macular hole closure in the early postoperative period using optical coherence tomography. Retina. 2000;20(3):232-7.

18. Saha N, Lake S, Wang BZ. Early imaging of a macular hole following vitrectomy with primary silicone oil tamponade. Clin Ophthalmol. 2011;5:1637-8.

19. Bayraktar S, Bayraktar Z, Yilmaz O. Influence of Scan Radius Correction for Ocular Magnification and Relationship Between Scan Radius With Retinal Nerve Fiber Layer Thickness Measured by Optical Coherence Tomography. J Glaucoma. 2001;10:163-9.

\section{Submit your next manuscript to BioMed Central and we will help you at every step:}

- We accept pre-submission inquiries

- Our selector tool helps you to find the most relevant journal

- We provide round the clock customer support

- Convenient online submission

- Thorough peer review

- Inclusion in PubMed and all major indexing services

- Maximum visibility for your research

Submit your manuscript at www.biomedcentral.com/submit 